\title{
Role of fortification and supplementation in achieving optimal biomarker status of B-vitamins for better mental health in older adults
}

\author{
K. Moore ${ }^{1}$, C.F. Hughes ${ }^{1}$, L. Hoey ${ }^{1}$, M. Ward ${ }^{1}$, K. Porter ${ }^{1}$, J.J. Strain ${ }^{1}$, K. Pentieva ${ }^{1}$, \\ A. Molloy ${ }^{2}$ and H. McNulty ${ }^{1}$ \\ ${ }^{1}$ Northern Ireland Centre for Food and Health, Ulster University, Coleraine, BT52 1SA and \\ ${ }^{2}$ Institute of Molecular Medicine, Trinity College, Dublin 2, Ireland
}

Worldwide the number of adults aged 60 years and over is predicted to reach 2 billion by $2050,{ }^{(1)}$ and hence the associated chronic diseases of ageing will continue to increase in the coming decades. Mental health disorders such as depression are common in older age and are major contributors to disability and poor quality of life. ${ }^{(2)}$ Folate and the related B-vitamins (vitamin B12, vitamin B6 and riboflavin) involved in one-carbon metabolism are required for normal brain function and thus may have a protective role against depression. We have recently reported that low biomarker status of the relevant B-vitamins was associated with an increased risk of depression: ${ }^{(3)}$ however it is unclear whether protective biomarker status can be achieved through natural food sources alone or if fortification and/or supplementation with B-vitamins is required. The aim of this study was to investigate the role of fortified food and supplements in optimising B-vitamin biomarker status in older adults, to levels associated with a reduced risk of depression.

This investigation was conducted as part of the Trinity Ulster Department of Agriculture (TUDA) Ageing cohort study ( $n$ 5186), where detailed health and lifestyle information was collected along with measurements of cognitive and mental health. Some $70 \%$ of participants were fortified food consumers while $11 \%$ were B-vitamin supplement users. Participants were categorised on the basis of dietary sources of B-vitamins as: natural food sources only; fortified food consumers; or supplement users (with and without concurrent consumption of fortified foods) (Table). B-vitamin biomarkers were examined in relation to each dietary category.

Data presented as medians. Differences were assessed using ANCOVA with Bonferroni post hoc tests on log-transformed data when applicable, controlling for age, gender, BMI and smoking. Values across a row without a common superscript letter are significantly different ( $\mathrm{P}$ < 0.05). Abbreviations: RBC, red blood cell; PLP, pyridoxal 5-phosphate; EGRac, erythrocyte glutathione reductase co-efficient.

\begin{tabular}{|c|c|c|c|c|c|}
\hline & \multirow[b]{2}{*}{ Natural Food Sources } & \multicolumn{3}{|c|}{ Fortified Food Consumer } & \multirow[b]{2}{*}{ Supplement User } \\
\hline & & Low & Medium & High & \\
\hline Servings/week & 0 & $1-4$ & $5-7$ & $8+$ & $0-8+$ \\
\hline TUDA Total $\%$ & 23.0 & $9 \cdot 5$ & $20 \cdot 7$ & 34.0 & $10 \cdot 8$ \\
\hline $\mathrm{RBC}$ folate $(\mathrm{nmol} / \mathrm{L})$ & $691^{\mathrm{a}}$ & $802^{\mathrm{b}}$ & $909^{\mathrm{c}}$ & $1138^{\mathrm{d}}$ & $1554^{\mathrm{e}}$ \\
\hline Serum Total Vitamin B12 (pmol/L) & $238^{\mathrm{a}}$ & $243^{\mathrm{ab}}$ & $260^{\mathrm{b}}$ & $271^{\mathrm{c}}$ & $293^{\mathrm{c}}$ \\
\hline Plasma Vitamin B6 PLP (nmol/L) & $47 \cdot 0^{\mathrm{a}}$ & $54 \cdot 1^{\mathrm{b}}$ & $60 \cdot 8^{\mathrm{c}}$ & $70 \cdot 3^{\mathrm{d}}$ & $70 \cdot 6^{d}$ \\
\hline Riboflavin status (EGRac) & $1 \cdot 35^{\mathrm{a}}$ & $1 \cdot 32^{\mathrm{b}}$ & $1 \cdot 28^{\mathrm{c}}$ & $1 \cdot 28^{\mathrm{c}}$ & $1 \cdot 24^{\mathrm{d}}$ \\
\hline Homocysteine $(\mu \mathrm{mol} / \mathrm{L})$ & $15 \cdot 2^{\mathrm{a}}$ & $13 \cdot 7^{\mathrm{b}}$ & $13 \cdot 7^{\mathrm{b}}$ & $12 \cdot 6^{\mathrm{c}}$ & $12 \cdot 2^{\mathrm{c}}$ \\
\hline
\end{tabular}

Biomarker status of each B-vitamin increased significantly with increasing intakes of fortified foods while non-consumers of fortified foods or supplements had the lowest status of all B-vitamin biomarkers. As previously reported, ${ }^{(3)}$ lowest biomarker status of folate ( $p$ 0.003), vitamin B6 ( $p$ 0.034) and riboflavin ( $p$ 0.011) were, in turn, associated with an increased risk of depression by $47-78 \%$

In conclusion, natural food sources alone appear to be insufficient in achieving a biomarker concentration of B-vitamins associated with lowest risk of depression, while regular consumption of fortified foods or supplements appear to be associated with biomarker concentrations that may protect mental health in ageing. These findings, if confirmed through randomised controlled trials, may have implications for dietary recommendations or fortification policy.

1. UN Department of Economic and Social Affairs/Population Division. (2015) New York: United Nations.

2. Prince M, Wu F, Guo Y, et al. (2015) Lancet, 385(9967), 549-562.

3. Moore K, Hughes C, Hoey L, et al. (2016) Proc Nutr Soc, 75, OCE3 E115. 